\title{
Alveolar proteinosis and nocardiosis: a patient treated by bronchopulmonary lavage
}

\author{
J. Pascual, M.A. Gómez Aguinaga, ${ }^{1}$ R. Vidal, A. Maudes, ${ }^{1}$ A. Sureda, E. Gómez \\ Mampaso $^{2}$ and L. Fogué ${ }^{3}$
}

Departments of Pulmonary Diseases, ${ }^{1}$ Intensive Care, ${ }^{2}$ Microbiology and ${ }^{3}$ Pathology, Hospital Ramón y Cajal, Madrid, Spain.

\begin{abstract}
Summary: Alveolar proteinosis is a relatively rare disease of unclear pathogenesis associated with opportunistic infections. Although nocardiosis is the most frequent one, only 22 cases have been reported previously and are reviewed here.

We present a patient with alveolar proteinosis with nocardiosis treated as an emergency with bilateral bronchopulmonary lavage and antibiotics. No previous cases of this association have been successfully managed in this way.
\end{abstract}

\section{Introduction}

Pulmonary alveolar proteinosis (PAP) is a rare disease characterized by the intra-alveolar accumulation of periodic acid-Schiff positive proteinaceous material rich in phospholipids. ${ }^{\prime}$

The pathogenesis of this disease remains unclear, although superimposed infections by unusual pathogens typically occur, Nocardia asteroides being the most common one. As far as we know, only 22 cases of this association have been previously reported. ${ }^{1-20}$

PAP usually has an indolent course. In the case we present, urgent bilateral bronchopulmonary lavage (BPL) was performed because of the rapid progression of the disease. This is the first case in which the PAP associated with nocardiosis has been managed with BPL.

\section{Case report}

The patient was a 37 year old bricklayer with a 3 month history of dyspnoea on exertion, cough with minimal production of mucoid sputum and loss of $10 \mathrm{~kg}$ in weight. One week before admission low-grade fever was noticed. He smoked a pack of cigarettes daily for 20 years. Examination revealed cyanosis, digital clubbing and basal crackles. The temperature was $37.8^{\circ} \mathrm{C}$ and the respirations were $26 /$ minute. The haemoglobin was $20 \mathrm{~g} / \mathrm{dl}$, the white cell count was $19 \times 10^{9} / 1$ and the lactic dehydrogenase was $764 \mathrm{IU} /$ litre. Arterial blood on room air at rest revealed $\mathrm{PaO}_{2}$

Correspondence: J. Pascual Santos, M.D., P. Florida, 31 3B, 28008 Madrid, Spain.

Accepted: 22 December 1988
$6 \mathrm{kPa}$ and $\mathrm{PaCO}_{2} 3.5 \mathrm{kPa}$, and $\mathrm{pH}$ 7.43. X-ray films of the chest showed diffuse bilateral alveolar infiltrates which spared the costophrenic angles. Diagnostic fibreoptic bronchoscopy with segmental lavage was performed. Flocculent material with Gram-positive filaments identified as Nocardia asteroides was obtained. Whitish disseminated lesions with erythematous margins were seen throughout the bronchial mucosa. Histological examination showed a distorted mucosa with fragmented and devitalized nocardias in it. The diagnosis of PAP was confirmed by transbronchial biopsy.

During the first week of hospitalization the patient's clinical and radiological situation (Figure 1) rapidly worsened. The $\mathrm{PaO}_{2}$ on $100 \%$ oxygen was $6.2 \mathrm{kPa}$. The patient was started on intravenous cotrimoxazole and a therapeutic BPL was performed on the left lung following a previously described technique ${ }^{21}$ using saline buffered to $\mathrm{pH} 7.8$. Three days later, the other lung was also lavaged. On the 15th hospital day, the patient was discharged almost symptom-free. Blood controls were normal and arterial blood disclosed that the $\mathrm{PaO}_{2}$ was $66 \mathrm{mmHg}(8.6 \mathrm{kPa})$, the $\mathrm{PaCO}_{2}$ $38 \mathrm{mmHg}(4.9 \mathrm{kPa})$ and the $\mathrm{pH} 7.40$, while the patient was breathing room air. X-ray films also showed complete clearing of the infiltration. The patient remains asymptomatic after a year on cotrimoxazole $1920 \mathrm{mg}$ twice daily.

\section{Discussion}

PAP has had a 'classic' association with Nocardia asteroides. Indeed, Preger ${ }^{22}$ reported that up to $10 \%$ of 


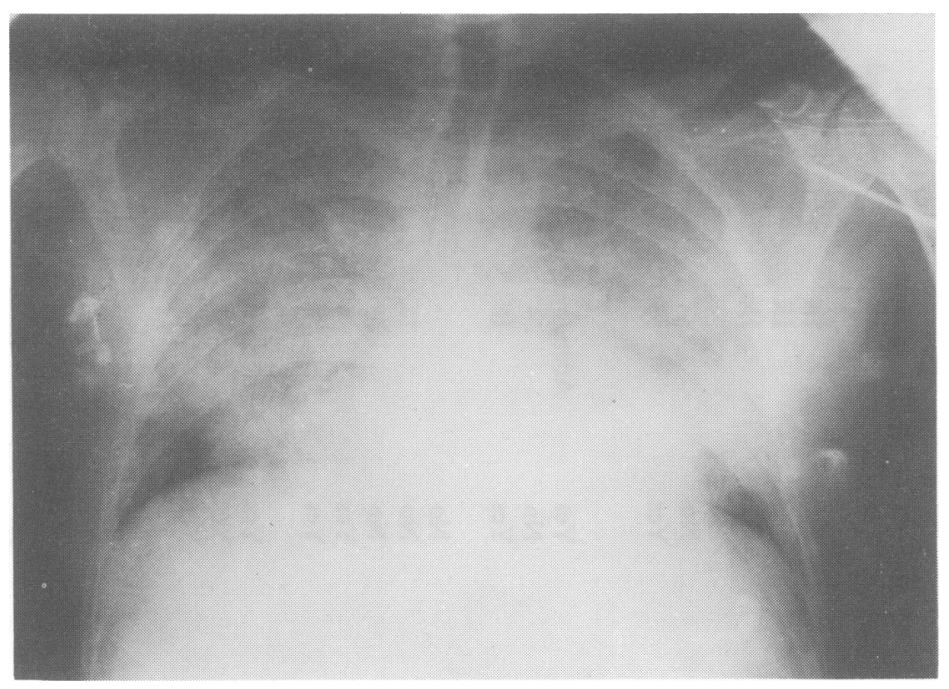

Figure 1 X-ray film of the chest showing extensive bilateral alveolar infiltrates.

cases of PAP were complicated by nocardiosis. Nevertheless, in the review of cases of nocardiosis by Palmer et $a .^{23}$ only $2 \%$ of the patients had PAP. To date, 400-500 cases of PAP have been reported and we have found only 23 associated with nocardiosis, including our own (Table I). Thus, from a pure statistical perspective PAP rarely underlies nocardiosis.

The average age of the patients was 40.8 years, range $22-58$ years and the male-female ratio was $2.8: 1$. The presentation and diagnosis of PAP and nocardiosis was simultaneous in only 7 cases. Usually, symptoms of PAP developed insidiously for months or years and then, fever, chills or other infectious manifestations supervene. When there was central nervous system (CNS) involvement, focal neurological features such as unilateral headache, seizures or hemiplegia were noted.

The morphological diagnosis of PAP was established by means of open lung biopsy in 14 cases. However, the diagnosis can be made safely by transbronchial lung biopsy and examination of the effluent material from selective bronchoalveolar lavage (BAL), ${ }^{24}$ as in our case. In one case only BAL was necessary; in 5 cases the diagnosis of PAP was made at necropsy, in one by transparietal biopsy and in the remaining one by lobectomy.

Nocardia species isolated were $N$. asteroides in 22 cases and $N$. brasiliensis in one. ${ }^{2}$ Pulmonary involvement was present in 19 cases and 6 of them had systemic lesions (skin, CNS, kidney or elbow). Pulmonary infection was predominant in the upper right lobe in 7 patients and was diffuse in the other 12. CNS was involved in 6 cases, 4 of them as an apparent primary infection. The cases in which cerebral nocar- diosis developed in persons without a pulmonary focus of infection are surprising: we can only assume that a small pulmonary focus went undiscovered.

Sputum Gram's stain was unremarkable except for 2 cases $^{14,20}$ and sputum culture established the diagnosis in 6 patients. In 4 patients bronchoscopy yielded a turbid fluid which grew Nocardia; in another case Nocardia was isolated from joint aspirate. ${ }^{17}$ The culture of the piece obtained at surgery yielded the microorganism in another case. ${ }^{19}$ In the cases with CNS involvement, the diagnosis was made by culturing the fluid obtained during brain abscess drainage. The remaining 7 patients were diagnosed as having nocardiosis at autopsy.

Only three patients were given corticosteroids before the diagnosis of nocardiosis was made. The relationship of steroid therapy, PAP and infection is not as important for nocardiosis as it may be for fungus infections. Pulmonary defence mechanisms in patients with PAP are altered, and in vitro studies have shown that alveolar macrophages in PAP are defective. ${ }^{25}$ González Rothi and Harris ${ }^{26}$ have recently confirmed that alveolar macrophages in PAP are dysfunctional and their finding of decreased phagolysosome fusion may be related to the high incidence of uncommon infections in these patients. These observations support the hypothesis that in patients with PAP, locally produced 'toxic' substances may impair alveolar clearance and contribute to the pathogenesis of this disease and superinfections.

Of the 23 patients reviewed 11 died and 12 were reported to be alive. In only 4 of the dead patients was the diagnosis of nocardiosis previously known. The infection was treated with antibiotics in 20 cases. 


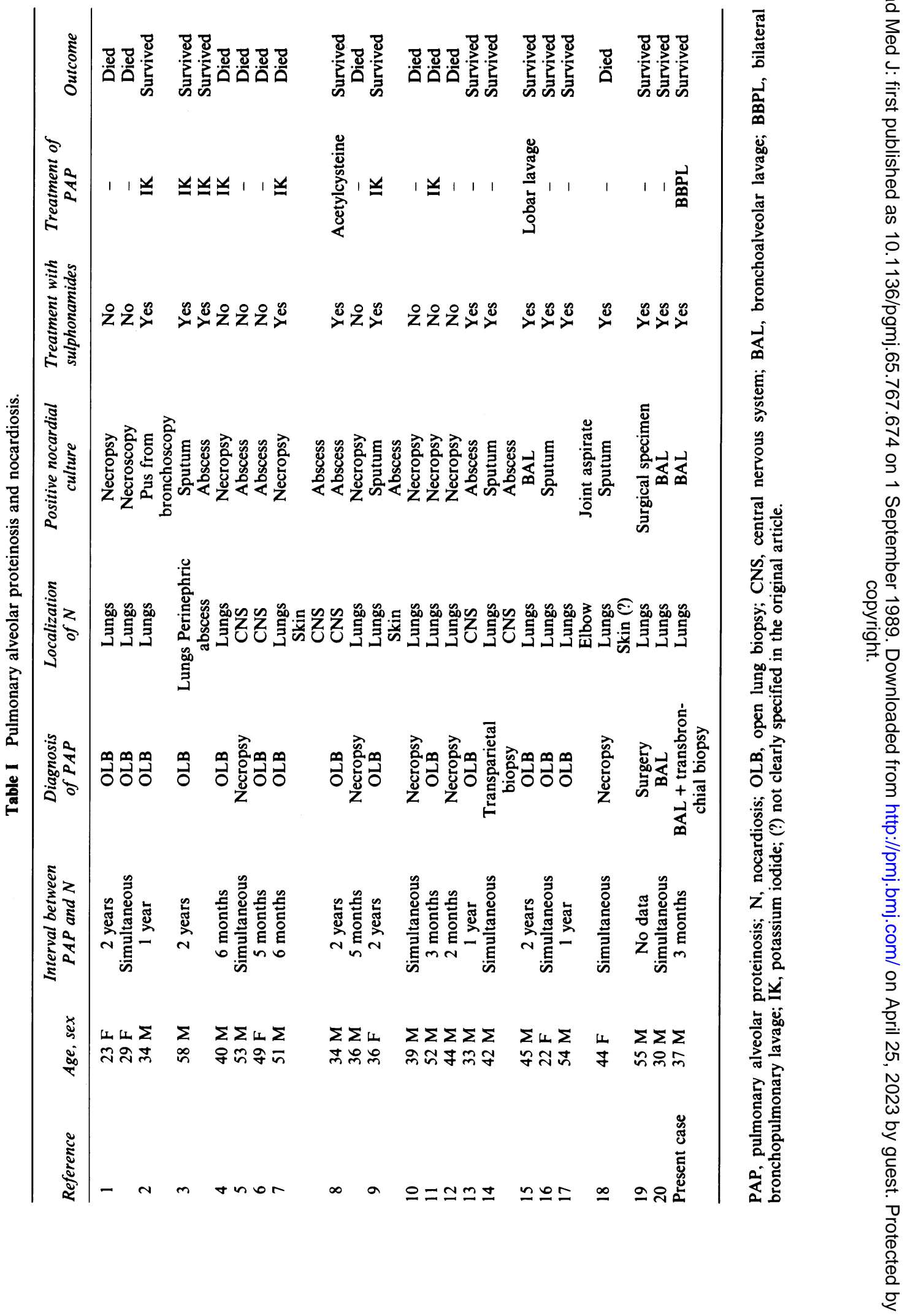


Sulphonamides and cotrimoxazole were the most useful agents; only two patients treated with them died. Cotrimoxazole is probably the current treatment of choice for nocardiosis. ${ }^{18,20}$

PAP usually has an indolent course; spontaneous resolution has been reported in one third of the cases described. ${ }^{27}$ PAP has a good prognosis in the long run $^{21,27,28}$ and usually is not a life-threatening disease. However, the severe cases generally need repeated BPL. PAP has not been treated with BPL in any case associated with nocardiosis. Only Harris et al. ${ }^{16}$ performed a localized lobar lavage in their case. Our patient, whose respiratory function rapidly deter-

\section{References}

1. Rosen, S.H., Castleman, B. \& Liebow, A.A. Pulmonary alveolar proteinosis. $N$ Engl $J$ Med 1958, 258: 1123-1142.

2. Burbank, B., Morrione, T.G. \& Cutler, S.S. Pulmonary alveolar proteinosis and nocardiosis. Am J Med 1960, 28: $1002-1007$.

3. Anderson, B.R., Ecklund, R.E. \& Kellow, W.F. Pulmonary alveolar proteinosis with systemic nocardiosis. JAMA 1960, 174: 110-113.

4. Raich, R.A., Casey, F. \& Hall, W.H. Pulmonary and cutaneous nocardiosis: the significance of the laboratory isolation of Nocardia. Am Rev Resp Dis 1961, 83: 505-509.

5. Saltzman, H.A., Chick, E.W. \& Cowant, N.F. Nocardiosis as complication of other diseases. Lab Invest 1962, 11: $1110-1117$.

6. Taleghani-Far, M., Barber, J.B., Simpson, C. \& Harden, K.A. Cerebral nocardiosis and alveolar proteinosis. $\mathbf{A m}$ Rev Resp Dis 1964, 89: 561-566.

7. Andriole, V.T., Balla, M. \& Wilson, G.L. The association of nocardiosis and pulmonary alveolar proteinosis: a case study. Ann Intern Med 1964, 60: 266-275.

8. Carlsen, T.T., Hill, R.B. \& Rowlands, D.T. Nocardiosis and pulmonary alveolar proteinosis. Ann Intern Med 1964, 60: 275-281.

9. Larson, R.K. \& Gordinier, R. Pulmonary alveolar proteinosis: report of six cases, review of the literature and formulation of a new theory. Ann Intern Med 1965, 62: $292-312$.

10. Martinez Maldonado, M., Arellano, G.R. Pulmonary alveolar proteinosis, nocardiosis and chronic granulocytic leukemia. South Med J 1966, 59: 901-905.

11. Summers, J.E. Pulmonary alveolar proteinosis. Calif Med 1966, 104: 428-436.

12. Boniver, J., Focan, C., Bury, J. \& Marcelle, R. Proteinose alveolaire et nocardiose. Etude anatomoclinique d'un cas. Conceptions pathogéniques actuelles. Acta Clin Belg 1973, 28: 162-175.

13. Supena, R., Karlin, D., Strate, R. \& Cramer, P.G. Pulmonary alveolar proteinosis and nocardia brain abscess: report of a case. Arch Neurol 1974, 30: 266-268.

14. Maderazo, G. \& Quintiliani, R. Treatment of nocardial infections with trimethoprim and sulfamethoxazole. Am J Med 1974, 57: 671-675. iorated was successfully managed with both cotrimoxazole and bilateral BPL. We can suggest that when opportunistic infections in PAP lead to significant respiratory failure, BPL should be performed without delay.

Probably, the prompt diagnosis of PAP and repeated BPL have made superinfections almost nonexistent. Only 5 cases of PAP and nocardiosis have been reported during the last decade ${ }^{16-20}$ and in three recent large series of PAP no infection has been superadded. ${ }^{21,27,28}$ Nevertheless, careful microbiological research is mandatory for patients with PAP.

15. Harris, J.O., Castle, J.R., Swenson, E.W. \& Block, A.J. Lobar lavage: therapeutic benefit in pulmonary alveolar filling disorders. Chest 1974, 65: 655-659.

16. Rubin, E., Weisbrod, G.L. \& Sanders, D.E. Pulmonary alveolar proteinosis. Relationship to silicosis and pulmonary infection. Radiology 1980, 135: 35-41.

17. Clague, H.W., Harth, M., Hellyer, D. \& Morgan, K.C. Septic arthritis due to Nocardia asteroides in association with pulmonary alveolar proteinosis. $J$ Rheumatol 1982 , 9: 469-472.

18. Case records of the Massachusetts General Hospital (case 19-1983). N Engl J Med 1983, 308: 1147-1156.

19. Smego, R.A., Moeller, M.B. \& Gallis, H.A. Trimethoprim-sulfamethoxazole therapy for Nocardia infections. Arch Intern Med 1983, 143: 711-722.

20. Case records of the Massachusetts General Hospital (Case 18-1988). N Engl J Med 1988, 318: 1186-1194.

21. Claypool, W.D., Rogers, R.M. \& Matuschak, G.M. Update on the clinical diagnosis, management and pathogenesis of pulmonary alveolar proteinosis (phospholipidosis). Chest 1981, 85: 550-558.

22. Preger, L. Pulmonary alveolar proteinosis. Radiology 1969, 92: $1291-1295$.

23. Palmer, D.L., Harvey, R.L. \& Wheeler, J.K. Diagnostic and therapeutic considerations in Nocardia asteroides infection. Medicine (Baltimore) 1974, 53: 391-401.

24. Rubinstein, I., Mullen, J.B.M. \& Hoffstein, V. Morphologic diagnosis of idiophatic pulmonary alveolar lipoproteinosis-revisited. Arch Intern Med 1988, 148: 813-816.

25. Nugent, K.M. \& Pesanti, E.L. Macrophage function in pulmonary alveolar proteinosis. Am Rev Resp Dis 1983, 127: $780-781$.

26. González Rothi, R.J. \& Harris, J.O. Pulmonary alveolar proteinosis. Further evaluation of abnormal alveolar macrophages. Chest 1986, 90: 656-661.

27. Prakash, U.B.S., Barham, S.S., Carpenter, H.A., Dines, D.E. \& Marsh, H.M. Pulmonary alveolar phospholipoproteinosis: experience with 34 cases and a review. Mayo Clin Proc 1987, 62: 499-518.

28. Kariman, K., Kylstra, J.A. \& Spock, A. Pulmonary alveolar proteinosis: prospective clinical experience in 23 patients for 15 years. Lung 1984, 162: 223-231. 